\title{
The outer membrane protein OprQ and adherence of Pseudomonas aeruginosa to human fibronectin
}

Correspondence

Cliff Boucher

jboucher@uttyler.edu

Received 6 August 2009

Revised 18 December 2009

Accepted 14 January 2010

\author{
Abraham Arhin and Cliff Boucher \\ The University of Texas at Tyler, 3900 University Blvd, Tyler, TX 75701, USA
}

\begin{abstract}
Outer membrane proteins of the Gram-negative organism Pseudomonas aeruginosa play a significant role in membrane permeability, antibiotic resistance, nutrient uptake, and virulence in the infection site. In this study, we show that the $P$. aeruginosa outer membrane protein OprQ, a member of the OprD superfamily, is involved in the binding of human fibronectin (Fn). Some members of the OprD subfamily have been reported to be important in the uptake of nutrients from the environment. Comparison of wild-type and mutant strains of $P$. aeruginosa revealed that inactivation of the oprQ gene does not reduce the growth rate. Although it does not appear to be involved in nutrient uptake, an increased doubling time was reproducibly observed with the loss of OprQ in $P$. aeruginosa. Utilizing an oprQ-xylE transcriptional fusion, we determined that the PA2760 gene, encoding OprQ, was upregulated under conditions of decreased iron and magnesium. This upregulation appears to occur in early exponential phase. Insertional inactivation of PA2760 in the $P$. aeruginosa wild-type background did not produce a significant increase in resistance to any antibiotic tested, a phenotype that is typical of OprD family members.

Interestingly, the in trans expression of OprQ in the $\triangle$ oprQ PAO1 mutant resulted in increased sensitivity to certain antibiotics. These findings suggest that OprQ is under dual regulation with other $P$. aeruginosa genes. Intact $P$. aeruginosa cells are capable of binding human $\mathrm{Fn}$. We found that loss of OprQ resulted in a reduction of binding to plasmatic Fn in vitro. Finally, we present a discussion of the possible role of the $P$. aeruginosa outer membrane protein OprQ in adhesion to epithelial cells, thereby increasing colonization and subsequently enhancing lung destruction by $P$. aeruginosa.
\end{abstract}

\section{INTRODUCTION}

Pseudomonas aeruginosa is an opportunistic Gram-negative pathogen that causes infections in individuals with altered immune systems, such as burn, HIV, nosocomial and neutropenic patients (DiGiandomenico et al., 2007; Sadikot et al., 2005). This pathogen is also notorious for its role in infections of the respiratory tract of patients with cystic fibrosis (Wagner \& Iglewski, 2008). The ability of P. aeruginosa to cause such a wide range of infections is, in part, due to the outer membrane proteins that it expresses (Wu et al., 2005). One major class of outer membrane proteins (Oprs), containing members that function as virulence factors, is the OprD superfamily (Tamber et al., 2006).

Outer membrane proteins are involved in many aspects of growth and development of the bacterial cell (Hancock \& Brinkman, 2002). The outer membrane protein OprD superfamily consists of 19 members, all of which display at least $46 \%$ similarity at the amino acid level (Tamber et al., 2006). The members of this family participate in amino acid and peptide transport, antibiotic uptake, and transport of

Abbreviation: Fn, fibronectin. carbon sources (Hancock \& Brinkman, 2002). In addition, the permeability of the $P$. aeruginosa outer membrane has been attributed, at least in part, to the surface expression of the OprD class of proteins (Strateva \& Yordanov, 2009). Although OprD homologues may perform multiple roles, it does not appear that the function of the channels of this family is non-specific. Each member of the OprD subfamily, at least those involved in nutrient uptake, seems to have a very narrow specificity (Tamber \& Hancock, 2003). For example, $P$. aeruginosa OprD, a homologue of the Escherichia coli porin $\mathrm{OmpF}$, exists in the outer membrane in a $\beta$-barrel configuration, and is known to play a significant role in the uptake of basic amino acids and resistance to imepenem and carbapenem (Hancock \& Brinkman, 2002; Lister, 2002). The exact role for some members, such as OprQ and OprI, has yet to be determined. Recently, the group of Tamber has reported that a specific nutrient substrate for OprQ and OprI cannot be identified, at least in the $P$. aeruginosa PAK background (Hancock \& Brinkman, 2002).

It has been reported that as many as 100 outer membrane proteins are produced by $P$. aeruginosa (Hancock \& Brinkman, 2002). Proteins localized to the outer membrane 
of $P$. aeruginosa have been shown not only to be involved in nutrient uptake and antibiotic resistance, but also to participate in this bacterium's pathogenicity. Some $P$. aeruginosa outer membrane virulence factors, such as XcpQ (Brok et al., 1999; Robert et al., 2005), XqhA (Martinez et al., 1998; Michel et al., 2007), EstA (Wilhelm et al., 1999), OprG (McPhee et al., 2009) and OprE (Yamano et al., 1998), have become areas of active research. However, there remain many Oprs that have not been investigated and their respective roles in $P$. aeruginosa have not yet been identified. One such potential virulence factor is the $P$. aeruginosa outer membrane protein OprQ. It has been reported that OprQ is a member of the OprD superfamily of proteins (Jaouen et al., 2006; Tamber et al., 2006). As stated above, initial studies of OprQ in $P$. aeruginosa did not reveal a specific role (Tamber et al., 2006). Studies of OprQ function in Pseudomonas fluorescens suggest that it plays a role significantly different from that of other members of the OprD subfamily. In this regard, OprQ in P. fluorescens has been shown to bind fibronectin (Fn) in vitro (RebièreHuët et al., 2002). The function of $P$. aeruginosa OprQ in growth, antibiotic resistance and the colonization of the host is currently not known, but these studies suggest that OprQ is directly involved in the disease process.

In this study, we report the unexpected finding that loss of OprQ resulted in an increased rate of growth. We also found that in the inactivated oprQ background, the overexpression of OprQ may increase $P$. aeruginosa sensitivity to antibiotics. In addition, we determined that OprQ is upregulated under conditions of low iron and magnesium. Since these conditions are thought to mimic conditions at known sites of $P$. aeruginosa infection, it is possible that OprQ is needed for the establishment of disease. Finally, we report that OprQ in $P$. aeruginosa is potentially involved in adhering to Fn, thus resulting in the initial colonization of the host epithelial tissue.

\section{METHODS}

Bacterial plasmids, strains and culture conditions. The strains, primers and plasmids used in this study are listed in Table 1. PAA2760 is a derivative of the P. aeruginosa strain PAO1. All strains were grown in the minimal medium M63, Pseudomonas Isolation Agar (PIA; Difco) or Luria-Bertani (LB; Difco) medium, with supplementation as indicated.

Using the PAO1 genomic database, oligonucleotides were designed to encompass the sequence between $P A 2760$ and the upstream gene PA2759. Primers 2760F-3 and 2760R-1 for the DNA sequence of PA2760 were used in a PCR to amplify the gene for cloning. The PCR product was then cloned into the vector PCR2.1 to create the plasmid pAA2760Pr using the TOPO TA cloning kit (Invitrogen). This plasmid was then digested with the restriction enzyme EcoRI (Invitrogen), and the $232 \mathrm{bp}$ promoter fragment was isolated and subsequently cloned into the EcoRI-digested vector pVDX18 (Konyecsni \& Deretic, 1988), creating the fusion plasmid pAA2760-XylE.

To generate the suicide vector pAA2760Tc, the primers $2760 \mathrm{~F}-1$ and 2760R-2 were used in a PCR to amplify a $1.5 \mathrm{~kb}$ fragment of the

Table 1. Bacterial strains, plasmids and primers

\begin{tabular}{|c|c|c|}
\hline Strain, primer or plasmid & Description & Source or reference \\
\hline \multicolumn{3}{|l|}{ P. aeruginosa strains } \\
\hline $\mathrm{PAO} 1$ & Wild-type prototroph & B. Holloway (Monash University, Australia) \\
\hline PAA2760 & PAO1 oprQ:: $\mathrm{Tc}^{\mathrm{r}}$ & This work \\
\hline \multicolumn{3}{|l|}{ E. coli strain } \\
\hline $\mathrm{DH} 5 \alpha$ & $\operatorname{lac} Z^{+}, \operatorname{rec}^{+}, \mathrm{Gal}^{+}$ & Invitrogen \\
\hline \multicolumn{3}{|l|}{ Primers } \\
\hline $2760 \mathrm{~F}-1$ & 5'-GCTCAGGAACAGGTCCT-3' & This work \\
\hline $2760 \mathrm{~F}-2$ & 5'-GGACGGTGGGCGTCGG-3' & This work \\
\hline $2760 \mathrm{~F}-3$ & 5'-GTTCTGGTATTGTTGCGCC-3' & This work \\
\hline 2760R-1 & 5'-GGAAACGACGAAGAACCAG-3' & This work \\
\hline $2760 \mathrm{R}-2$ & 5'-CGGGCACAACGCGACC-3' & This work \\
\hline 2760R-3 & 5'-CCAAGAGCAGCGCAAGC-3' & This work \\
\hline \multicolumn{3}{|l|}{ Plasmids } \\
\hline pRK2013 & $\mathrm{tra}^{+}, \mathrm{mob}^{+}, \mathrm{Kan}^{\mathrm{r}}$ & D. R. Helinski, University of California, San Diego \\
\hline pUC19 & $\operatorname{lac} Z \alpha^{+}$, ColE1, $\mathrm{Ap}^{\mathrm{r}}$ & New England Biolabs \\
\hline pVDX18 & IncQ, $\mathrm{Ap}^{\mathrm{r}}, \mathrm{mob}^{+}, x y l E^{+}$ & Konyecsni \& Deretic (1988) \\
\hline pVDtac39 & IncQ, $\mathrm{mob}^{+}, \mathrm{tac}, \mathrm{lacl}^{q}$ & Deretic et al. (1987) \\
\hline pUCTc & pUC19, $\mathrm{Ap}^{\mathrm{r}}$, tet $^{+}$ & Martin et al. (1993) \\
\hline pAA2760 & pCR2.1, partial oprQ coding sequence & This work \\
\hline pAA2760Tc & pUC19 oprQ:: $\mathrm{Tc}^{\mathrm{r}}$ & This work \\
\hline pAAOprQ & pCR2.1, complete $o p r Q$ coding sequence & This work \\
\hline pAA2760WT & pVDtac39 oprQ ${ }^{+}$ & This work \\
\hline pAA2760Pr & pCR2.1, OprQ promoter region & This work \\
\hline pAA2760-XylE & pVDX18 oprQ: : xylE & This work \\
\hline
\end{tabular}


PA2760 gene. This fragment was cloned into plasmid pCR2.1 (Invitrogen), creating plasmid pAA2760. This plasmid was doubledigested with the restriction enzymes HindIII and XbaI (both from New England Biolabs), and the fragment containing the PA2760 gene was extracted from an agarose gel via a PureLink Quick Gel Extraction kit (Invitrogen). The plasmid vector pUC19 (New England Biolabs) was also double-digested with HindIII/XbaI and subsequently ligated with the PA2760 HindIII/XbaI fragment. E. coli strain DH5 $\alpha$, harbouring the newly constructed pUC2760 plasmid, was grown on agar (Difco plates containing $50 \mu \mathrm{g}$ ampicillin $\mathrm{ml}^{-1}$ (Invitrogen). The isolated pUC2760 plasmid DNA was further digested with the restriction enzyme BstEII (Invitrogen), purified and blunt-ended with large fragment Klenow (Invitrogen). The plasmid pUCTc, containing a cassette that confers tetracycline resistance $\left(\mathrm{Tc}^{\mathrm{R}}\right)$, was digested with PstI. The $1.5 \mathrm{~kb}$ tetracycline resistance gene cassette was isolated from a $1 \%$ agarose gel and bluntended. The vector ( $\mathrm{pUC2760}$ ) and insert $\left(\mathrm{Tc}^{\mathrm{R}}\right.$ cassette) were ligated in the presence of $5 \%$ PEG 1000 in order to concentrate the blunt-ended DNA fragments. Following ligation, $\mathrm{DH} 5 \alpha$ cells were transformed with the ligated DNA and plated onto LB agar plates containing $10 \mu \mathrm{g}$ tetracycline $\mathrm{ml}^{-1}$ (Invitrogen), and the suicide vector pAA2760Tc containing the tetracycline insert was identified via digestion with HindIII and XbaI and visualization on a $1 \%$ agarose gel.

For complementation of the OprQ mutant, we created the plasmid pAA2760WT. Using the primers $2760 \mathrm{~F}-1$ and $2760 \mathrm{R}-3$ in a PCR, we amplified the complete coding region of the oprQ gene. The amplified gene was cloned into the pCR2.1 vector (Invitrogen). The wild-type sequence of the PCR fragment was verified by sequencing using the Beckman CEQ 8000 Genetic Analysis System (Beckman Coulter). The newly created plasmid pAAOprQ was digested with EcoRI, and the $1.9 \mathrm{~kb}$ oprQ gene fragment was gel-purified and cloned into the EcoRI-digested plasmid pVDtac-39 (Deretic et al., 1987).

All bacterial triparental conjugations were carried out using the donor E. coli DH5 $\alpha$ strain carrying the plasmids pAA2760-XylE and pAA2760WT with conjugates grown in LB supplemented with either ampicillin $\left(50 \mu \mathrm{g} \mathrm{ml}^{-1}\right)$ or tetracycline $\left(10 \mu \mathrm{g} \mathrm{ml}^{-1}\right)$, respectively. Recipient PAO1 was grown in LB and E. coli harbouring the helper plasmid pRK2013 (Figurski \& Helinski, 1979) in LB with kanamycin $\left(50 \mu \mathrm{g} \mathrm{ml}^{-1}\right)$. Each strain was grown overnight in $5 \mathrm{ml}$ of medium, spun, washed in sterile saline and resuspended in $0.3 \mathrm{ml} \mathrm{LB}$ medium. The Pseudomonas recipient strain, donor DH5 $\alpha$, and the helper E. coli were combined $(0.9 \mathrm{ml}$ total $)$ and passed through a $0.45 \mu \mathrm{m}$ pore-size filter. The filter was placed on an LB plate and grown overnight at $37{ }^{\circ} \mathrm{C}$. The bacterial growth from the filter was plated on PIA plates with either $1000 \mu \mathrm{g}$ carbenicillin $\mathrm{ml}^{-1}$ or $150 \mu \mathrm{g}$ tetracycline $\mathrm{ml}^{-1}$. Growth of $P$. aeruginosa strains for doubling time determination was performed using both LB and M63 media supplemented with $1 \%$ glucose or glycerol (as carbon source), and either $0.2 \%$ potassium nitrate or ammonium sulfate (as nitrogen source). c.f.u. were determined by serial dilution in $0.8 \%$ sodium chloride and plating on LB media. Growth of $P$. aeruginosa strains analysed for xylE promoter activity was performed using M63 medium or M63 medium containing either $\mathrm{FeCl}_{3}$ or $\mathrm{MgSO}_{4}$ supplement, as indicated. All $P$. aeruginosa strains were grown to an $\mathrm{OD}_{600}$ of 1.5 and 1 for iron and magnesium conditions, respectively. Upon reaching the appropriate $\mathrm{OD}_{600}$, the $P$. aeruginosa cells were spun, washed and assayed as described below.

Electroporation and generation of the insertional mutant PAA2760. The newly created pAA2760Tc plasmid was used via electroporation to transform the wild-type P. aeruginosa strain PAO1 using the method of Smith \& Iglewski (1989). Briefly, to create electrocompetent $P$. aeruginosa cells, $500 \mu \mathrm{l}$ of $300 \mathrm{mM}$ sucrose was added to a microcentrifuge tube and approximately $3 \mathrm{mg}$ of PAO1 cells, grown overnight in $\mathrm{LB}$ at $37^{\circ} \mathrm{C}$, was suspended in the sucrose solution. The $P$. aeruginosa cells were washed twice, and the final pellet was resuspended in $40 \mu \mathrm{l} 300 \mathrm{mM}$ sucrose. The electroporation-competent $P$. aeruginosa was kept on ice for the remainder of the procedure. Five microlitres of pAA2760Tc DNA was added to the PAO1 cell pulsed at $3.0 \mathrm{kV} \mathrm{mm}^{-1}$ using a Bio-Rad Electropulser, $1 \mathrm{ml}$ of LB broth was added, and the cells were incubated at $37^{\circ} \mathrm{C}$ with shaking for $1 \mathrm{~h}$. Cells were then plated $(100 \mu \mathrm{l})$ onto LB agar plates containing $50 \mu \mathrm{g}$ tetracycline $\mathrm{ml}^{-1}$ and incubated overnight at $37{ }^{\circ} \mathrm{C}$. Possible PA2760 knockout mutants were screened by replica plating on LB agar plates containing $500 \mu \mathrm{g}$ carbenicillin $\mathrm{ml}^{-1}$. Since the pUC19 plasmid is non-replicative in PAO1, sensitivity to carbenicillin $\left(\mathrm{Carb}^{\mathrm{S}}\right)$ and resistance to tetracycline $\left(\mathrm{Tet}^{\mathrm{R}}\right)$ were used to ensure that the oprQ gene was knocked out during electroporation. Carbenicillin sensitivity indicates the loss of the pUC19 plasmid. The PAO1 $2760::$ Tet $^{\mathrm{r}} \mathrm{Carb}^{\mathrm{s}}$ conjugant was verified for proper insertion of the genetic cassette by PCR using the primers 2760F-2 and 2760R-2, which flank the tetracycline insertion site.

XyIE assays. The catechol-2,3-dioxygenase (XylE) activity of the OprQ transcriptional fusion was assayed as described by Schweizer \& Hoang (1995). Three separate protein extracts were prepared by growing conjugated cells overnight in M63 medium supplemented with $300 \mu \mathrm{g}$ carbenicillin $\mathrm{ml}^{-1}$. One millilitre of a $P$. aeruginosa overnight culture was used to inoculate a fresh solution of M63 in a $1: 100$ dilution, and the culture was grown at $37^{\circ} \mathrm{C}$ with shaking until the appropriate $\mathrm{OD}_{600}$ was reached (see above). The concentrations of magnesium and iron used for each experimental condition were as follows: (i) regular magnesium, $1.0 \mathrm{mM} \mathrm{MgSO}_{4} \cdot 7 \mathrm{H}_{2} \mathrm{O}$; (ii) low magnesium, $0.2 \mathrm{mM} \mathrm{MgSO}_{4} \cdot 7 \mathrm{H}_{2} \mathrm{O}$; (iii) regular iron, $0.1 \mathrm{mM} \mathrm{FeCl}$; and (iv) low iron, $0.01 \mathrm{mM} \mathrm{FeCl}_{3}$. All cultures were centrifuged at 10000 r.p.m. for $10 \mathrm{~min}$. The cells were then resuspended in $5 \mathrm{ml}$ lysis buffer $(50 \mathrm{mM}$ potassium phosphate buffer, $\mathrm{pH} 7.5,10 \%$ acetone; Sigma-Aldrich). Cells were sonicated with a Branson cell sonifier for $1 \mathrm{~min}$ on ice with no interruptions. The disrupted cells were centrifuged cold and protein concentration was determined by the Bradford method in a Beckman Coulter spectrophotometer at $375 \mathrm{~nm}$. Samples were prepared by adding to a $3 \mathrm{ml}$ cuvette (BioRad) the following: $2.5 \mathrm{ml} \mathrm{H}_{2} \mathrm{O}, 0.3 \mathrm{ml} 0.5 \mathrm{M}$ potassium phosphate buffer and $0.1 \mathrm{ml} 10 \mathrm{mM}$ catechol, all at room temperature. The correct amount of extract was added to equilibrate samples to $30 \mu \mathrm{g}$ $\mathrm{ml}^{-1}$, except the blank, which received no protein. Samples were added immediately and inverted two to three times to mix thoroughly. Readings were done at $375 \mathrm{~nm}$, for $150 \mathrm{~s}$ with a $2.5 \mathrm{~s}$ interval. For each condition, regular and low iron or magnesium, a total of three independent sample protein extracts were analysed.

Antibiotic disk diffusion assay. Disks saturated separately with cefotaxime $(30 \mu \mathrm{g})$, gentamicin $(10 \mu \mathrm{g})$, amoxicillin/clavulanic acid $(30 \mu \mathrm{g})$, erythromycin $(15 \mu \mathrm{g})$, vancomycin $(30 \mu \mathrm{g})$, levofloxacin $(5 \mu \mathrm{g})$, ampicillin $(10 \mu \mathrm{g})$, piperacillin $(100 \mu \mathrm{g})$, chloramphenicol $(2 \mu \mathrm{g})$, neomycin $(30 \mu \mathrm{g})$, meropenem $(10 \mu \mathrm{g})$ and aztreonam $(30 \mu \mathrm{g})$ were applied to Mueller-Hinton agar plates containing $P$. aeruginosa strain PAO1, PAA2760 or PAA2760 carrying wild-type oprQ. The plates were incubated for $18 \mathrm{~h}$ at $37^{\circ} \mathrm{C}$. At the end of the incubation period, the diameters $(\mathrm{mm})$ of the zones of growth inhibition were measured. Replicates for antibiotic susceptibility determination were performed for each strain using separate inoculations from individual cultures.

Determination of MIC. The MICs of colistin, ciproflaxin, imipenem monohydrate, tetracycline and tobramycin sulfate at starting concentrations of $16,10,10,50$ and $4 \mu \mathrm{g} \mathrm{m}^{-1}$, respectively, were determined by twofold serial dilution using a sterile 96-well flatbottomed microtitre plate, as described by Andrews (2001). The MICs of each individual strain were determined from at least three independent assays. 
Fn-binding assay. Fn-binding assays were performed in 96-well sterile microtitre plates. Wells were coated overnight at $8{ }^{\circ} \mathrm{C}$ with $1.25 \mu \mathrm{g}$ plasmatic Fn (Sigma-Aldrich) in sterile PBS (125 $\mu$ l per well of $10 \mu \mathrm{g} \mathrm{Fn} \mathrm{ml} \mathrm{m}^{-1}$ ). The wells were washed four times with $125 \mu \mathrm{l}$ of $1 \%(\mathrm{w} / \mathrm{v})$ BSA in PBS. Then wells were blocked with $125 \mu \mathrm{l}$ of $1 \%$ (w/v) BSA in PBS for $1 \mathrm{~h}$. Just before adding bacteria, wells were washed six times with sterile prewarmed PBS.

P. aeruginosa strains PAO1, PAA2760 and PAA2760 pAA2760WT were grown overnight at $37^{\circ} \mathrm{C}$ on $\mathrm{LB}$ plates. Bacteria were resuspended in PBS and collected by centrifugation. The bacteria were washed twice with prewarmed PBS and resuspended in the same sterile buffer at $\mathrm{OD}_{600} 0.4$. A total of $50 \mu$ of bacterial suspension was added to the Fn-coated wells and incubated at room temperature for $3 \mathrm{~h}$. Non-adherent bacteria were removed by washing six times, using vigorous pipetting, with sterile $\mathrm{PBS}$. Adherent bacteria were then collected with $100 \mu$ sterile PBS containing $0.5 \%$ Triton X-100 under manual desorption. Bacteria were plated by 10 -fold serial dilutions onto LB plates, incubated overnight at $37{ }^{\circ} \mathrm{C}$, and quantified the following day. A minimum of three replicates for Fn binding were performed for each strain using separate inoculations from individual cultures.

Quantification of pyocyanin. For quantification of pyocyanin levels in PAO1, PAA2760 and PAA2760 carrying the wild-type oprQ in trans, strains were grown in LB medium for $48 \mathrm{~h}$ at $30{ }^{\circ} \mathrm{C}$. Cultures were centrifuged at $7000 \mathrm{~g}$ for $15 \mathrm{~min}$. The pyocyanin pigment was extracted using chloroform/0.2 $\mathrm{M} \mathrm{HCl}(1: 1, \mathrm{v} / \mathrm{v})$. Quantification was performed by the measurement of $A_{530}$ on a Beckman DU 800 spectrophotometer (Beckman Coulter).

Statistical analysis. Statistical analysis of the results of the growth analysis, $x y l E$ assay, antibiotic susceptibility and Fn binding were conducted by analysis of variance (Tukey's post test) using Systat (Systat Software).

\section{RESULTS}

\section{Growth rate increase upon loss of OprQ}

The OprD family of porins is diverse, with a few members participating in nutrient acquisition from the environment (Hancock \& Brinkman, 2002; Tamber \& Hancock, 2003). As a member of this OprD subfamily, it is possible that the $P$. aeruginosa OprQ participates in nutrient uptake. One earlier study using a transposon oprQ mutant in the $P$. aeruginosa PAK background was unable to determine a specific amino acid transported by PA2760 (Tamber et al., 2006). It remains possible that OprQ is involved in the overall growth of $P$. aeruginosa. To further investigate the growth phenotype for OprQ in the $P$. aeruginosa PAO1 wild-type background, we insertionally inactivated the oprQ gene using a tetracycline resistance gene cassette. The parent and mutant strains were grown on minimal M63 medium to determine whether any phenotypic changes could be observed. Interestingly, $P$. aeruginosa strain PAA2760, in which OprQ expression was disrupted, had a significant increase in growth rate as compared with the PAO1 parent when cultured in LB medium (Fig. 1). As seen in Fig. 1, the faster doubling time for PAA2760, $29.9 \mathrm{~min}$, was retarded back to the wild-type rate of $44 \mathrm{~min}$ when pAA2760WT was introduced into the OprQ mutant.
Substitution of glucose and potassium nitrate with glycerol and ammonium sulfate resulted in the same growth phenotype, suggesting that the increased doubling time is independent of the carbon or nitrogen source. These results indicate that OprQ is not required for the uptake of essential nutrients for growth. Futhermore, the observation of an increased growth rate suggests that OprQ is involved, directly or indirectly, in the regulation of other proteins/ porins that are important in $P$. aeruginosa growth.

\section{Transcription and expression of OprQ is increased under stress conditions}

Under conditions of poor oxygenation, Jaouen et al. (2006) revealed, using RT-PCR, that the $P$. fluorescens oprQ gene is overexpressed. Oxygen stress and the induction of an OprD family outer membrane protein are not without precedents. It has been reported that OprE in P. aeruginosa is similarly induced in oxygen-depleted environments (Yamano et al., 1993). In order to identify other potential stressors that may induce the expression of the oprQ gene, the PA2760 promoter region, an intergenic sequence containing a potential promoter-binding site of the stress response sigma factor $\mathrm{RpoH}$, was cloned into a $x y l E$ vector to analyse the expression of OprQ under varying conditions, such as that which might be encountered in the infection site. Comparisons were made between PAO1, harbouring the pAA2760-XylE plasmid, grown in M63 medium supplemented with regular magnesium $\left(1.0 \mathrm{mM} \mathrm{MgSO}_{4} .7 \mathrm{H}_{2} \mathrm{O}\right.$ ) and iron $\left(0.1 \mathrm{mM} \mathrm{FeCl}_{3}\right)$ and $\mathrm{M} 63$ medium supplemented with low magnesium $\left(0.2 \mathrm{mM} \mathrm{MgSO}_{4} .7 \mathrm{H}_{2} \mathrm{O}\right)$ and iron $\left(0.01 \mathrm{mM} \mathrm{FeCl}_{3}\right)$. Exposure to conditions of low magnesium resulted in a fivefold increase in transcription of the oprQ gene (Fig. 2). An even greater effect on promoter activity was observed when the strains were placed under low-iron stress. Under this condition, a 13-fold increase in oprQ promoter activity was observed (Fig. 2). These results suggest that OprQ is required for survival under conditions where $P$. aeruginosa is exposed to adverse growth conditions.

\section{OprQ and antibiotic resistance}

The ability of $P$. aeruginosa to be resistant to such a wide range of antibiotics is due to the outer membrane porins that it produces (Strateva \& Yordanov, 2009). In particular, members of the OprD outer membrane protein superfamily have been shown to function in antibiotic resistance (Tamber et al., 2006). Since OprQ does not appear to play a significant role in growth, and given the potential role in antibiotic resistance, we hypothesized that OprQ affects the sensitivity of $P$. aeruginosa to these antimicrobials. It has been reported that OprQ is not important for carbapenem resistance (Okamoto et al., 1999). Therefore, we expanded this analysis of $P$. aeruginosa sensitivity upon inactivation of the $o p r Q$ gene to include 18 different antibiotics (gentamicin, neomycin, amoxicillin/clavulanic acid, ampicillin, piperacillin, levoflaxin, ciproflaxin, meropenem, imipenem, cefsulodin, cefotaxime, colistin, vancomycin, tetracycline, 


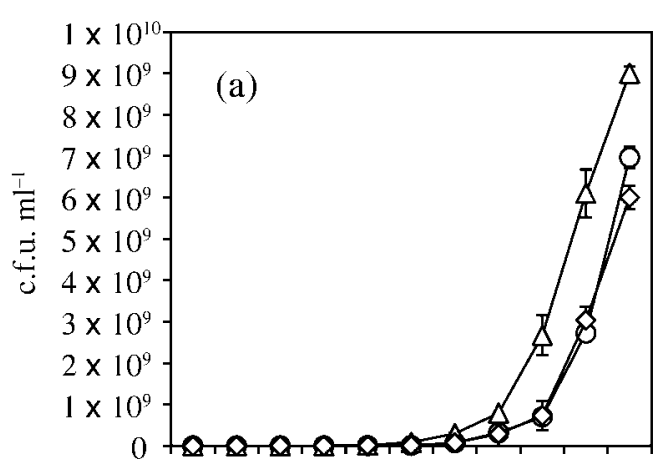

$\begin{array}{lllllllllll}1 & 2 & 3 & 4 & 5 & 6 & 7 & 8 & 9 & 10 & 11\end{array}$

Time (h)

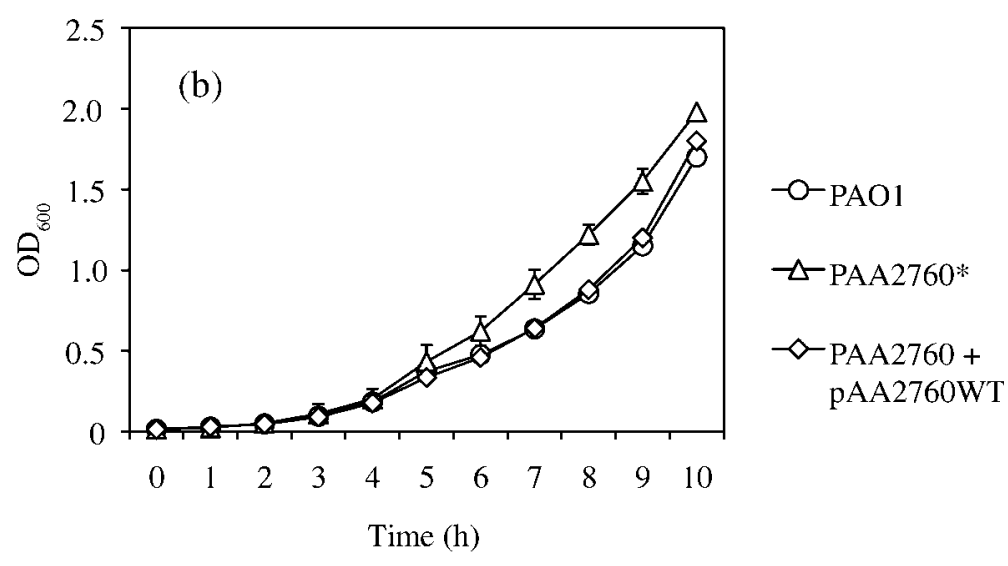

Fig. 1. Growth curve of PAO1 wild-type $(\bigcirc)$, PAA2760 (oprQ::Tcr) $(\triangle)$ and PAA2760 harbouring the plasmid pAA2760WT $\left(o p r Q^{+}\right)(\diamond)$. (a) Strains grown at $37{ }^{\circ} \mathrm{C}$ in LB medium. (b) Strains grown at $37{ }^{\circ} \mathrm{C}$ in M63 minimal medium supplemented with $1 \%$ glucose and $0.2 \%$ potassium nitrate. Asterisks indicate statistical difference between PAO1/ PAA2760 harbouring pAA2760WT and PAA2760 as determined by Tukey's post test (ANOVA). tobramycin, chloramphenicol, erythromycin and aztreonam) from 12 different classes (aminoglycosides, penicillins, quinolones, carbepenems, cephalosporins, polymyxins, glycopeptides, tetracyclines, aminoglycosides, amphenicols, macrolides and monobactams). Analysis of susceptibilities of the wild-type, PAA2760 and PAA2760 harbouring the pAA2760WT plasmid strains to these antibiotics was performed using the Kirby-Bauer and MIC methods (Andrews, 2001; Traub \& Leonhard, 1994). Comparison of the zone of inhibition on disk diffusion for PAO1 and PAA2760 showed no significant statistical difference (Fig. 3). Interestingly, it was observed that when the PAA2760 strain expressed wild-type OprQ, from the pAA2760WT plasmid, there was a reproducible increase in sensitivity to gentami-

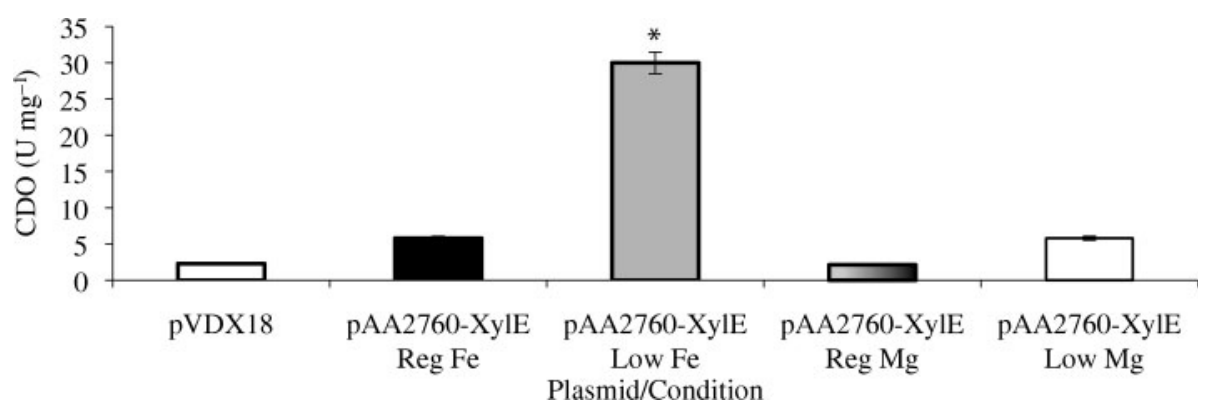

Fig. 2. Transcriptional activation of the $P$. aeruginosa oprQ gene under stress conditions. The units of catechol-2,3dioxygenase (CDO) are specific activity per milligram of protein. Bacterial growth in $\mathrm{M} 63$ was supplemented with regular iron (0.1 $\mathrm{mM} \mathrm{FeCl}_{3}$; Reg Fe), low iron (0.01 mM FeCl 3 ; Low Fe), regular magnesium (1.0 mM MgSO $\mathrm{mg}_{2} \mathrm{O} ; \mathrm{Reg} \mathrm{Mg}$ ) or low magnesium $\left(0.2 \mathrm{mM} \mathrm{MgSO}{ }_{4} \cdot 7 \mathrm{H}_{2} \mathrm{O}\right.$; Low $\mathrm{Mg}$ ) as indicated. The statistical significance for regular versus low iron was $P=0.001$. An asterisk indicates statistical significance for regular versus low magnesium as determined by ANOVA (Tukey's post test, SYSTAT). 
cin, neomycin and meropenem (Fig. 3). When we assayed for antibiotic resistance using the MIC method, the same sensitivity phenotype was observed for the antibiotics colistin, imipenem, tetracycline and tobramycin when wild-type OprQ was placed in trans (Fig. 4). This suggests that increased expression and outer membrane localization of OprQ lead to the repression of proteins required for $P$. aeruginosa resistance to these antibiotics.

\section{Determination of Fn-binding activity}

The binding of human Fn and its relationship to infection for outer membrane proteins of $P$. fluorescens have been established. A total of six P. fluorescens Fn-binding outer membrane proteins (FnBPs) have been identified (RebièreHuët et al., 2002). One of these proteins, identified as a $44 \mathrm{kDa}$ outer membrane protein, is homologous to the $P$. aeruginosa outer membrane protein OprQ (Jaouen et al., 2006). Studies presented here have excluded OprQ from a role in nutrient uptake and resistance to many classes of antibiotics. However, it remains possible that OprQ is indirectly involved in $P$. aeruginosa sensitivity to certain antibiotics. Therefore, based on the reports of $P$. fluorescens and the similarity of OprQ to these FnBPs, we hypothesized that OprQ could play a role in $P$. aeruginosa binding to the extracellular matrix via Fn. In order to test the ability of $P$. aeruginosa to bind plasmatic Fn, we compared wild-type PAO1 with the PAO1 $\Delta o p r Q$ strain PAA2760. Addition of whole-cell $P$. aeruginosa PAO1 to plates coated with human Fn resulted in adsorption (Fig. 4). Upon addition of PAA2760 to Fn in vitro, there was a significant drop, 88fold, in the ability of $P$. aeruginosa to adhere to the extracellular matrix protein (Fig. 4). The introduction of the plasmid pAA2760WT into PAA2760 led to a 41-fold

(a)

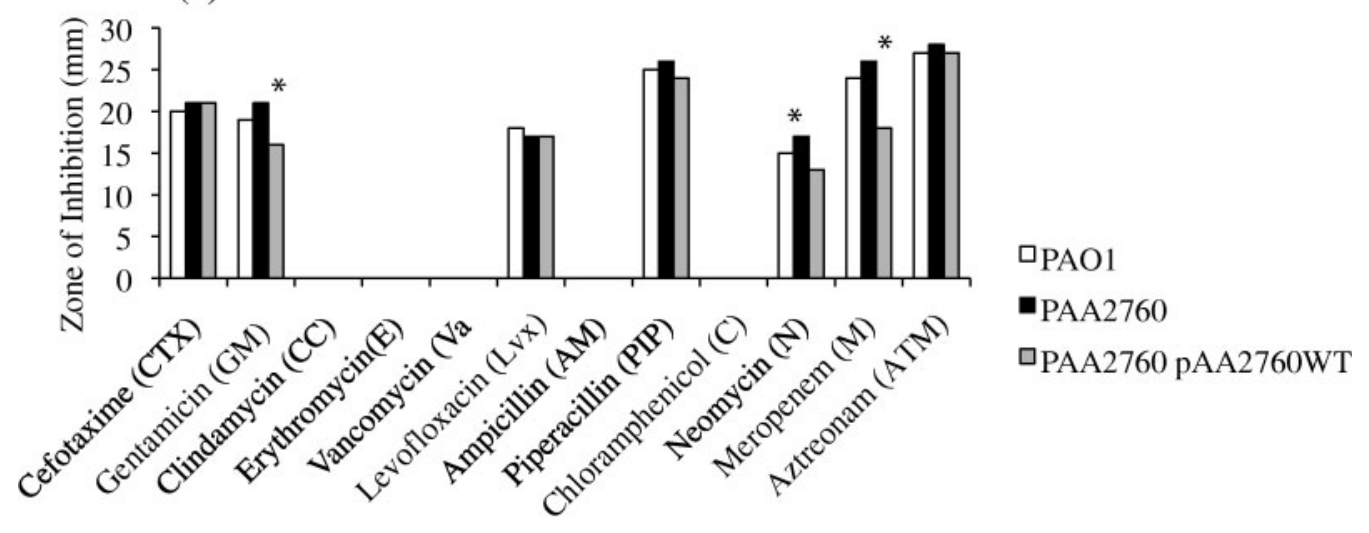

Antibiotic

(b)

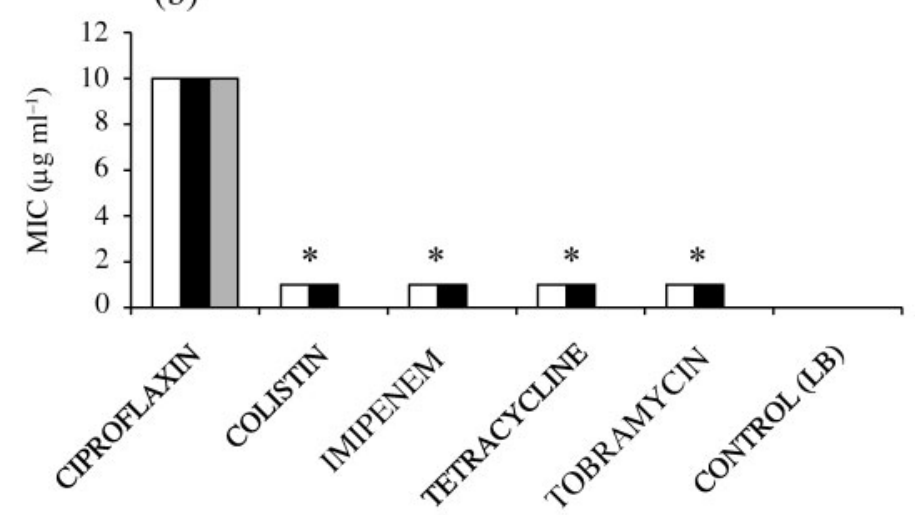

Antibiotic
口PAOI

-PAA2760

口PAA2760 pAA2760WT

Fig. 3. $P$. aeruginosa strains $P A O 1, P A A 2760(\Delta o p r Q)$ and PAA2760 pAA2760WT (oprQ ${ }^{+}$) and the effect of antibiotics on growth. (a) Kirby-Bauer disk diffusion assay; cells were grown overnight at $37^{\circ} \mathrm{C}$ in LB medium, standardized using the 0.5 McFarland standard, and spread onto Mueller-Hinton agar plates. (b) MIC; $P$. aeruginosa strains were grown in LB broth overnight at $37{ }^{\circ} \mathrm{C}$ with shaking. The growth density was adjusted to equal the $0.5 \mathrm{McF}$ arland standard and cells were incubated with antibiotic for $18 \mathrm{~h}$ at $37^{\circ} \mathrm{C}$. Asterisks indicate statistical significance between PAO1/PAA2760 and PAA2760 + pAA2760WT, as determined by ANOVA (Tukey's post test, SYSTAT). 


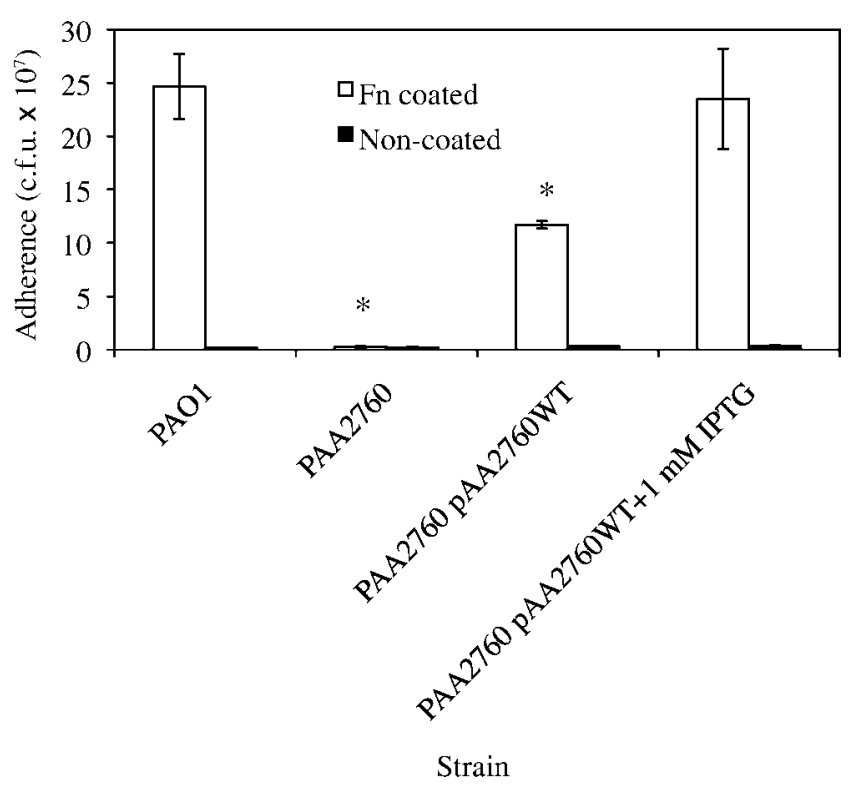

Fig. 4. Effect of OprQ on P. aeruginosa binding to human Fn. Binding of $P$. aeruginosa to $\mathrm{Fn}$ is reported as c.f.u. per well. The statistical significance between PAO1 and PAA2760 was $P=0.0153$. Overexpression of the oprQ gene was achieved by addition of $1 \mathrm{mM}$ IPTG to the growth medium. This concentration of IPTG was maintained during incubation of PAA2760 harbouring the pAA2760WT plasmid with Fn. Asterisks indicate statistical significance between PAO1 and PAA2760 as determined by ANOVA (Tukey's post test, SYSTAT).

increase in adsorption as compared with PAA2760 alone (Fig. 4). The restored binding to human Fn in vitro was still significantly lower than that observed for the $P$. aeruginosa parent strain PAO1. The complementation plasmid pAA2760WT contains an IPTG-inducible promoter. Addition of $1 \mathrm{mM}$ IPTG to PAA2760 carrying the pAA2760WT plasmid resulted in complete restoration of the binding by $P$. aeruginosa to levels indistiguishable from those of PAO1 (Fig. 4). These results suggest that OprQ is important in binding of the extracellular matrix protein Fn. Therefore, it is expected that this outer membrane protein will prove to be important for $P$. aeruginosa in causing disease. Futhermore, the level of adsorption to human Fn is determined by the amount of OprQ expressed by $P$. aeruginosa.

\section{DISCUSSION}

In this study, we provide evidence that the $P$. aeruginosa OprQ protein, a member of the OprD superfamily, does not function in roles common to this class of outer membrane proteins. The regulation of $o p r Q$ expression may be in dual regulation with other proteins, possibly Oprs that are specifically involved in nutrient uptake and resistance to antibiotics, since the PAO1 $\Delta$ oprQ strain displays an increased growth rate under stress conditions.
Furthermore, it was observed that the overexpression of OprQ could lead to increased sensitivity to certain classes of antibiotics. This suggests that expression of OprQ could be tied to the regulation of optimal growth characteristics for $P$. aeruginosa that favour the prevailing environmental condition. A model has been proposed for the outer membrane protein OmpC in E. coli whereby its transcriptional activation is reciprocal to that of the ompF gene (Ozawa \& Mizushima, 1983). Early studies described this regulatory phenotype as being closed. Under conditions that favour upregulation of the E. coli ompC gene, OmpF expression is decreased (Schnaitman \& McDonald, 1984). Likewise, when $o m p F$ transcription is activated there is a reciprocal repression of the ompC gene. This phenotype is also apparent when a mutation in $\mathrm{OmpF}$ is created in E. coli. The expression of OmpC in this background is derepressed (Ozawa \& Mizushima, 1983). It is now understood that this reciprocal regulation is controlled by the transcriptional factor OmpR (Yoshida et al., 2006). It has been speculated that this type of gene regulation could be widely utilized by prokaryotes (Schnaitman \& McDonald, 1984). Likewise, the increased sensitivity to certain antibiotics may be controlled at the transcriptional level. Seven clinical isolates of $P$. aeruginosa from a hospital in the USA have been found to overexpress the mexXY genes (Wolter et al., 2004). In all seven cases, the transcription of the oprD gene was repressed, resulting in a decrease in outer membrane OprD (Wolter et al., 2004). The data presented here seem to suggest that the regulation and expression of OprQ are similar to those of the systems that control OmpC and OprD. Upon entrance into the infection site, stress conditions encountered by $P$. aeruginosa may lead to an increased expression of OprQ and enhanced binding to host tissue via Fn. This would be in conjunction with a decrease in growth rate, a phenotype that could aid in this organism's ability to survive in the host.

The effect of OprQ on the expression of $P$. aeruginosa proteins may extend beyond those involved in metabolism, growth and antibiotic sensitivity. In addition to causing the effects previously discussed, OprQ appears to have an effect on the production of $P$. aeruginosa pyocyanin. Previous studies on pyocyanin have revealed that this secreted phenazine is important in $P$. aeruginosa disease. It has been associated with sepsis in burns (Muller et al., 2009), damage to ciliary function (Lau et al., 2004), apoptosis (Usher et al., 2002), inactivation of vacuolar ATPase (Ran et al., 2003) and damage to lung tissue (Lau et al., 2004). In both acute and chronic models of respiratory infection, Lau et al. (2004) demonstrated that $P$. aeruginosa virulence was maximal only with the expression of pyocyanin. This is in keeping with a recent finding that $P$. aeruginosa pyocyanin inhibits the respiratory dual oxidase-thiocyanate-lactoperoxidase system, thus resulting in a reduced killing of $P$. aeruginosa by the human bronchial epithelial cells and increased survival in the host lung (Rada et al., 2008). Inactivation of the oprQ gene in P. aeruginosa strain PAO1 yielded an increase in pyocyanin production (data not shown). As shown in Table 2, quantification of 
Table 2. Pyocyanin production (in $A_{530}$ units) and the OprO mutant phenotype

\begin{tabular}{|llll|}
\hline Strain & \multicolumn{1}{c}{ Plasmid } & \multicolumn{1}{c|}{ Genotype } & Pyocyanin \\
\hline PAO1 & None & Wild-type & $0.05 \pm 0.002$ \\
PAA2760 & None & PAO1 oprQ:: Tc & $0.15 \pm 0.010$ \\
PAA2760 & pAA2760WT & PAO1 oprQ:: $\mathrm{Tc}^{\mathrm{r}}$ oprQ & $0.03 \pm 0.001$ \\
\hline
\end{tabular}

pyocyanin from PAA2760 by $A_{530}$ revealed at least a threefold increase over $P$. aeruginosa PAO1 (0.05 vs 0.15 ). Data presented here seem to suggest that the early expression of OprQ on the outer membrane of $P$. aeruginosa may be important for colonization. However, upon establishing disease and the formation of an environment that favours diminished expression of OprQ, pyocyanin production may be increased. This would allow for the expression of the full complement of virulence factors and the establishment of more substantial disease. Further investigation into the relationship between OprQ and pyocyanin production will be required to determine the exact interaction.

Most pathogens have been reported to bind host tissue via the protein Fn, which aids in colonization and infection (Joh et al., 1999; Rebière-Huët et al., 1999). The expression of Fn in epithelial cells has been positively correlated with the degree of injury in affected tissues (Roman et al., 2006). It is possible that the damaged cells of patients that express high levels of Fn provide a means for $P$. aeruginosa to adhere to and colonize the patient. In addition, it has been demonstrated that in the host respiratory epithelium, the innate mechanisms that protect against invading pathogens can be compromised when levels of iron are altered (Ganz, 2009; Porto \& De Sousa, 2007). It is thought that the decreased level of iron in the respiratory tract is important as an innate mechanism against infection (Ganz, 2009). In this environment, for a true opportunistic pathogen such as $P$. aeruginosa, the ability to overcome the iron-reduced condition is crucial. The upregulation of virulence factors, especially in the initial stage of infection, would aid in $P$. aeruginosa colonization in this stressful host environment. This hypothesis is in agreement with the induction of the oprQ gene as presented in this study. We have determined that under conditions of reduced iron and magnesium, such as those described above, OprQ expression increases. Upon activation of oprQ, $P$. aeruginosa adherence to the host extracellular matrix would be enhanced. This phase of colonization to host tissue would lead to further tissue damage and the subsequent increased expression of Fn and thus more $P$. aeruginosa binding. This suggests that OprQ would be an important virulence factor involved in $P$. aeruginosa infections.

\section{ACKNOWLEDGEMENTS}

This research was supported by grant BOUCHERUTT3 from The University Of Texas at Tyler faculty research program.

\section{REFERENCES}

Andrews, J. M. (2001). Determination of minimum inhibitory concentrations. J Antimicrob Chemother 48 (suppl. 1), 5-16.

Brok, R., Van Gelder, P., Winterhalter, M., Ziese, U., Koster, A. J., de Cock, H., Koster, M., Tommassen, J. \& Bitter, W. (1999). The Cterminal domain of the Pseudomonas secretin XcpQ forms oligomeric rings with pore activity. J Mol Biol 294, 1169-1179.

Deretic, V., Chandrasekharappa, S., Gill, J. F., Chatterjee, D. K. \& Chakrabarty, A. M. (1987). A set of cassettes and improved vectors for genetic and biochemical characterization of Pseudomonas aeruginosa. Gene 57, 61-72.

DiGiandomenico, A., Rao, J., Harcher, K., Zaidi, T. S., Gardner, J., Neely, A. N., Pier, G. B. \& Goldberg, J. B. (2007). Intranasal immunization with heterologously expressed polysaccharide protects against multiple Pseudomonas aeruginosa infections. Proc Natl Acad Sci U S A 104, 4624-4629.

Figurski, D. H. \& Helinski, D. R. (1979). Replication of an origincontaining derivative of plasmid RK2 dependent on a plasmid function provided in trans. Proc Natl Acad Sci U S A 76, 1648-1652.

Ganz, T. (2009). Iron in innate immunity: starve the invaders. Curr Opin Immunol 21, 63-67.

Hancock, R. E. \& Brinkman, F. S. (2002). Function of pseudomonas porins in uptake and efflux. Annu Rev Microbiol 56, 17-38.

Jaouen, T., Coquet, L., Marvin-Guy, L., Orange, N., Chevalier, S. \& De, E. (2006). Functional characterization of Pseudomonas fluorescens OprE and OprQ membrane proteins. Biochem Biophys Res Commun 346, 1048-1052.

Joh, D., Wann, E. R., Kreikemeyer, B., Speziale, P. \& Hook, M. (1999). Role of fibronectin-binding MSCRAMMs in bacterial adherence and entry into mammalian cells. Matrix Biol 18, 211-223.

Konyecsni, W. M. \& Deretic, V. (1988). Broad-host-range plasmid and M13 bacteriophage-derived vectors for promoter analysis in Escherichia coli and Pseudomonas aeruginosa. Gene 74, 375-386.

Lau, G. W., Ran, H., Kong, F., Hassett, D. J. \& Mavrodi, D. (2004). Pseudomonas aeruginosa pyocyanin is critical for lung infection in mice. Infect Immun 72, 4275-4278.

Lister, P. D. (2002). Chromosomally-encoded resistance mechanisms of Pseudomonas aeruginosa: therapeutic implications. Am J Pharmacogenomics 2, 235-243.

Martin, D. W., Holloway, B. W. \& Deretic, V. (1993). Characterization of a locus determining the mucoid status of Pseudomonas aeruginosa: $\mathrm{AlgU}$ shows sequence similarities with a Bacillus sigma factor. J Bacteriol 175, 1153-1164.

Martinez, A., Ostrovsky, P. \& Nunn, D. N. (1998). Identification of an additional member of the secretin superfamily of proteins in Pseudomonas aeruginosa that is able to function in type II protein secretion. Mol Microbiol 28, 1235-1246.

McPhee, J. B., Tamber, S., Bains, M., Maier, E., Gellatly, S., Lo, A., Benz, R. \& Hancock, R. E. (2009). The major outer membrane protein OprG of Pseudomonas aeruginosa contributes to cytotoxicity and forms an anaerobically regulated, cation-selective channel. FEMS Microbiol Lett 296, 241-247.

Michel, G. P., Durand, E. \& Filloux, A. (2007). XphA/XqhA, a novel GspCD subunit for type II secretion in Pseudomonas aeruginosa. J Bacteriol 189, 3776-3783.

Muller, M., Li, Z. \& Maitz, P. K. (2009). Pseudomonas pyocyanin inhibits wound repair by inducing premature cellular senescence: role for p38 mitogen-activated protein kinase. Burns 35, 500-508.

Okamoto, K., Gotoh, N., Tsujimoto, H., Yamada, H., Yoshihara, E., Nakae, T. \& Nishino, T. (1999). Molecular cloning and characteriza- 
tion of the oprQ gene coding for outer membrane protein OprE3 of Pseudomonas aeruginosa. Microbiol Immunol 43, 297-301.

Ozawa, Y. \& Mizushima, S. (1983). Regulation of outer membrane porin protein synthesis in Escherichia coli $\mathrm{K}-12$ : ompF regulates the expression of ompC. J Bacteriol 154, 669-675.

Porto, G. \& De Sousa, M. (2007). Iron overload and immunity. World J Gastroenterol 13, 4707-4715.

Rada, B., Lekstrom, K., Damian, S., Dupuy, C. \& Leto, T. L. (2008). The Pseudomonas toxin pyocyanin inhibits the dual oxidase-based antimicrobial system as it imposes oxidative stress on airway epithelial cells. J Immunol 181, 4883-4893.

Ran, H., Hassett, D. J. \& Lau, G. W. (2003). Human targets of Pseudomonas aeruginosa pyocyanin. Proc Natl Acad Sci U S A 100, 14315-14320.

Rebière-Huët, J., Di Martino, P., Gallet, O. \& Hulen, C. (1999). Interactions of the Pseudomonas aeruginosa outer membrane proteins with plasma fibronectins. Bacterial adhesin investigation. C R Acad Sci III 322, 1071-1080.

Rebière-Huët, J., Guérillon, J., Pimenta, A. L., Di Martino, P., Orange, N. \& Hulen, C. (2002). Porins of Pseudomonas fluorescens MFO as fibronectin-binding proteins. FEMS Microbiol Lett 215, 121-126.

Robert, V., Filloux, A. \& Michel, G. P. (2005). Role of XcpP in the functionality of the Pseudomonas aeruginosa secreton. Res Microbiol 156, 880-886.

Roman, J., Rivera, H. N., Roser-Page, S., Sitaraman, S. V. \& Ritzenthaler, J. D. (2006). Adenosine induces fibronectin expression in lung epithelial cells: implications for airway remodeling. Am J Physiol Lung Cell Mol Physiol 290, L317-L325.

Sadikot, R. T., Blackwell, T. S., Christman, J. W. \& Prince, A. S. (2005). Pathogen-host interactions in Pseudomonas aeruginosa pneumonia. Am J Respir Crit Care Med 171, 1209-1223.

Schnaitman, C. A. \& McDonald, G. A. (1984). Regulation of outer membrane protein synthesis in Escherichia coli K-12: deletion of ompC affects expression of the OmpF protein. J Bacteriol 159, 555-563.

Schweizer, H. P. \& Hoang, T. T. (1995). An improved system for gene replacement and $x y l E$ fusion analysis in Pseudomonas aeruginosa. Gene 158, 15-22.

Smith, A. W. \& Iglewski, B. H. (1989). Transformation of Pseudomonas aeruginosa by electroporation. Nucleic Acids Res 17, 10509.
Strateva, T. \& Yordanov, D. (2009). Pseudomonas aeruginosa - a phenomenon of bacterial resistance. J Med Microbiol 58, 1133-1148.

Tamber, S. \& Hancock, R. E. (2003). On the mechanism of solute uptake in Pseudomonas. Front Biosci 8, s472-s483.

Tamber, S., Ochs, M. M. \& Hancock, R. E. (2006). Role of the novel OprD family of porins in nutrient uptake in Pseudomonas aeruginosa. $J$ Bacteriol 188, 45-54.

Traub, W. H. \& Leonhard, B. (1994). Agar disk diffusion (BauerKirby) tests with various fastidious and nonfastidious reference (ATCC) strains: comparison of several agar media. Chemotherapy $\mathbf{4 0}$, 374-383.

Usher, L. R., Lawson, R. A., Geary, I., Taylor, C. J., Bingle, C. D., Taylor, G. W. \& Whyte, M. K. (2002). Induction of neutrophil apoptosis by the Pseudomonas aeruginosa exotoxin pyocyanin: a potential mechanism of persistent infection. J Immunol 168, 18611868.

Wagner, V. E. \& Iglewski, B. H. (2008). P. aeruginosa biofilms in CF Infection. Clin Rev Allergy Immunol 35, 124-134.

Wilhelm, S., Tommassen, J. \& Jaeger, K. E. (1999). A novel lipolytic enzyme located in the outer membrane of Pseudomonas aeruginosa. J Bacteriol 181, 6977-6986.

Wolter, D. J., Smith-Moland, E., Goering, R. V., Hanson, N. D. \& Lister, P. D. (2004). Multidrug resistance associated with mexXY expression in clinical isolates of Pseudomonas aeruginosa from a Texas hospital. Diagn Microbiol Infect Dis 50, 43-50.

Wu, L., Estrada, O., Zaborina, O., Bains, M., Shen, L., Kohler, J. E., Patel, N., Musch, M. W., Chang, E. B. \& other authors (2005). Recognition of host immune activation by Pseudomonas aeruginosa. Science 309, 774-777.

Yamano, Y., Nishikawa, T. \& Komatsu, Y. (1993). Cloning and nucleotide sequence of anaerobically induced porin protein E1 (OprE) of Pseudomonas aeruginosa PAO1. Mol Microbiol 8, 993-1004.

Yamano, Y., Nishikawa, T. \& Komatsu, Y. (1998). Involvement of the RpoN protein in the transcription of the oprE gene in Pseudomonas aeruginosa. FEMS Microbiol Lett 162, 31-37.

Yoshida, T., Qin, L., Egger, L. A. \& Inouye, M. (2006). Transcription regulation of $o m p F$ and $o m p C$ by a single transcription factor, OmpR. J Biol Chem 281, 17114-17123.

Edited by: P. van der Ley 